\title{
Time-Reversal Ground-Penetrating Radar: Range Estimation With Cramér-Rao Lower Bounds
}

\author{
Foroohar Foroozan, Student Member, IEEE, and Amir Asif, Senior Member, IEEE
}

\begin{abstract}
In this paper, first, a new range-estimation technique using time reversal (TR) for ground-penetrating-radar (GPR) applications is presented. The estimator is referred to as the TR/GPR range estimator. The motivation for this paper comes from the need of accurately estimating the location of underground objects such as landmines or unexploded ordinance for safe clearance. Second, the Cramér-Rao lower bound (CRLB) for the performance of the TR/GPR range estimator is derived and compared with the CRLB for the conventional matched filter (MF). The CRLB analysis shows that the TR/GPR range estimator has the potential to achieve higher accuracy in estimating the location of the target than that of the conventional MF estimator. Third, the proposed TR/GPR estimator is tested using finite-difference time-domain simulations, where the surface-based reflection GPR is modeled using an electromagnetic transverse-magnetic (TM) mode formulation. In our simulations, the TR/GPR estimator outperforms the conventional MF approach by up to 5-dB reduction in mean square error at signal-to-noise ratios ranging from -20 to $20 \mathrm{~dB}$ for dry-soil environments.
\end{abstract}

Index Terms-Cramér-Rao bounds, ground-penetrating radar (GPR), multipath, range estimation, time reversal (TR).

\section{INTRODUCTION}

G ROUND-PENETRATING radar (GPR) is a geophysical approach that uses radar pulses in the UHF/VHF band of the radio spectrum for high-resolution imaging of shallow subsurfaces. Recently, GPR has been used extensively in civil engineering applications (for nondestructive testing of solid structures and pavements, locating buried objects, and studying soils) [1], [2], in environmental remediation (for defining landfills, contaminant plumes, and other remediation sites), and in archeology (for mapping archaeological features and cemeteries) [3]. A GPR system consists of a pair of transmitting and receiving antenna arrays, with the transmitting antennas probing the channel and the receiving antennas recording (and/or processing) the backscatter of the transmitted signals. The type of antennas, choice of the probing signal, and signalprocessing methods used for range estimation depend upon the application for which the GPR system is being designed [4]. Of interest in our research is the application of GPR to rangeestimation problems where the location of an underground object is to be estimated. Specifically, we are interested in developing time-reversal (TR) signal-processing techniques to

Manuscript received July 21, 2009; revised March 6, 2010. Date of publication June 14, 2010; date of current version September 24, 2010.

The authors are with the Department of Computer Science and Engineering, York University, Toronto, ON M3J 1P3, Canada (e-mail: asif@ cse.yorku.ca).

Color versions of one or more of the figures in this paper are available online at http://ieeexplore.iee.org.

Digital Object Identifier 10.1109/TGRS.2010.2047726

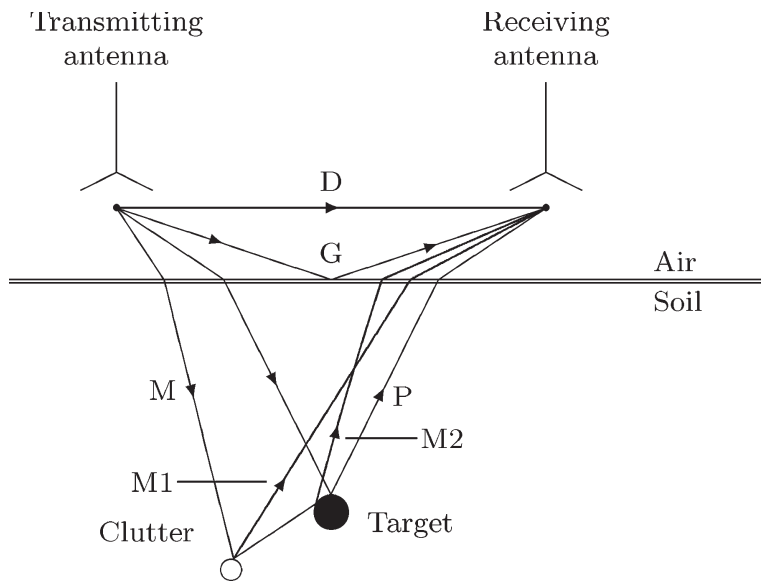

Fig. 1. Schematic drawing of a typical GPR system with multipath.

detect and estimate the location of an underground landmine or unexploded ordinance for safe clearance [5].

Fig. 1 shows a typical GPR system where a known waveform is transmitted from a single antenna element and the backscatter from the target is used to estimate the location of the target. Uncertainty exists because of observation noise, multipath, and clutter; the last two factors make it difficult to accurately determine the true sources of reflections. Possible sources of multipath are reflections from the Earth's surface (Path G) and clutter (Paths M1 and M2), which distort the backscatter reflection (Path P) received from the target. Note that Path M1 represents the straight-line path from the clutter to the receiver, while Path M2 interacts with the target on its way to the receiver. Path D is the directly coupled path ${ }^{1}$ between the transmitting and receiving antennas. In order to estimate the range of a buried target, signal-processing techniques such as background subtraction [4] and clutter suppression [6] are applied to extract the target reflected signal from the overall received signal. In this paper, we use the background subtraction procedure that eliminates the signals received from Paths G, M1, and D. Since Path M2 interacts with the target, it cannot be completely removed from the processed received signal by background subtraction. We note that the relationship between the clutter multipath signals and the target reflected signal in GPR is a function of many other parameters including the depth and dielectric properties of the underground object and its surrounding soil. Fig. 1 is an illustrative representation

\footnotetext{
${ }^{1}$ We follow the signal-processing terminology and refer to the straight line (Path D in Fig. 1) connecting the transmitter and receiver as the directly coupled path. In comparison, the shortest path (Path $\mathrm{P}$ ) connecting the transmitter, target, and receiver is referred to as the direct path.
} 


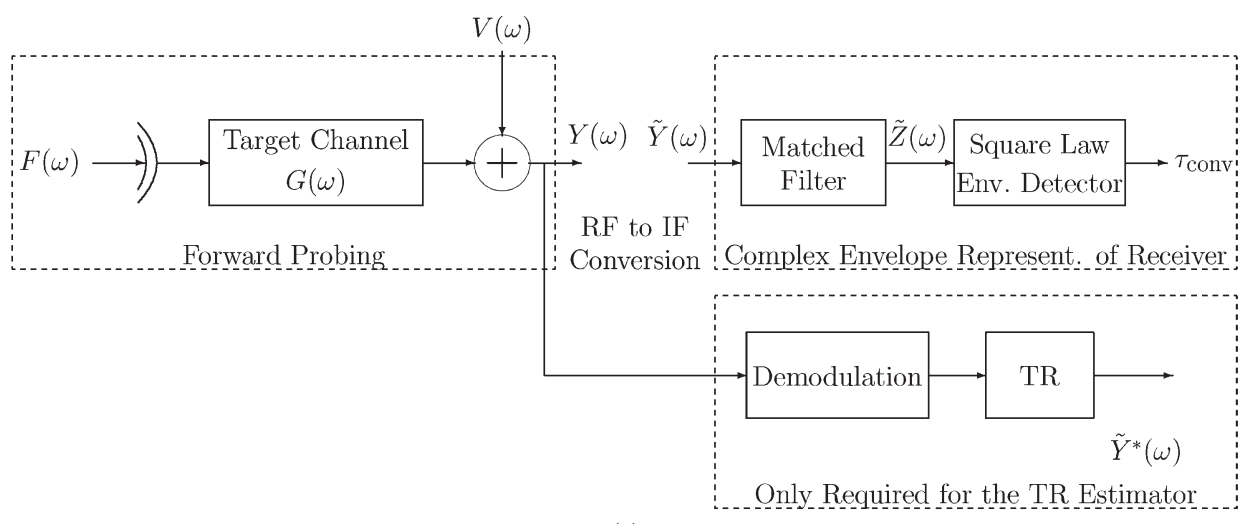

(a)

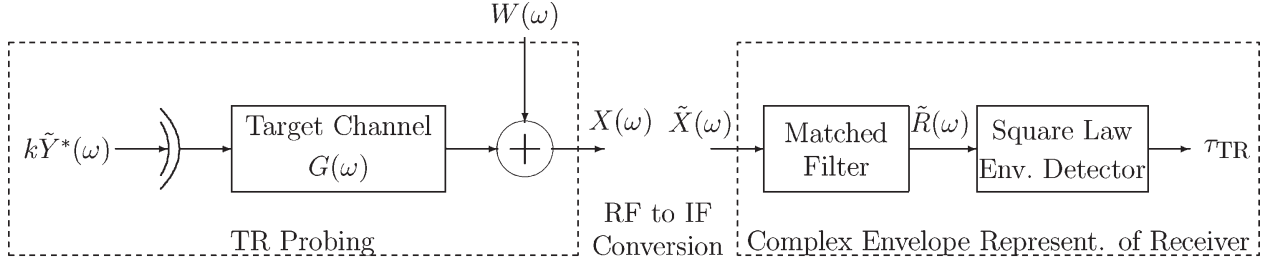

(b)

Fig. 2. Block diagrams representing the conventional and TR-based estimators for GPR applications. (a) Conventional time-delay estimator and (b) TR timedelay estimator. Signals shown in the figure in uppercase letters are FTs of their time-domain counterparts expressed in lowercase. $F(\omega)$, for example, is the FT of $f(t)$ and similarly for other signals.

of some of these multipaths. Additional multipaths originate from other sources such as the lower saturated layers of the earth. When high clutter is present, conventional GPR signalprocessing approaches typically fail to accurately estimate the location of the buried target.

This paper presents a new TR-based GPR range estimator for locating passive underground objects buried in a highclutter environment. The TR/GPR estimator consists of two observation stages. In Stage 1, a reference signal probes the medium and its backscatter $\tilde{y}^{(1)}(t)$ from the scattering elements is collected by the receiving antenna. The observation $\tilde{y}^{(1)}(t)$ is typically used by the conventional GPR range estimators. In TR [7], [8], a second step (Stage 2) is introduced, where the signal $\tilde{y}^{(1)}(t)$ observed during Stage 1 is energy normalized, time reversed, and retransmitted back into the medium. The backscatter of the time-reversed signal, obtained from this second TR stage, is used for estimating the location of the buried target in the proposed TR/GPR estimator. For detection and imaging applications, it has been shown previously [9]-[11] that TR adapts well to high-clutter environments with rich multipath. The authors of [11]-[14] extend the principle of TR to the electromagnetic domain. The first contribution of this paper is to extend the current state of art in electromagnetic TR to GPR range-estimation applications, where the emergence of software-driven waveform generators [15] provides us with the ability to modify the transmitted waveform to match the environment and makes TR reshaping in GPR a practically feasible approach. Second, we derive the Cramér-Rao lower bound (CRLB) for the performance of the TR/GPR range estimator and compare it with the CRLB for the conventional matched-filter (MF) approach for a simplified multipath propagation model (similar to Fig. 1). Third, we verify our theoretical analysis through simulations based on the finite-difference time-domain (FDTD) method
[16] and quantify the performance of the proposed TR/GPR range estimator. In our FDTD simulations, the TR/GPR provides a gain [reduction in mean square error (MSE)] of up to $5 \mathrm{~dB}$ for signal-to-noise ratios (SNRs) within -20 to $20 \mathrm{~dB}$ in dry-soil environments. The analytical models are derived for a dispersion-free environment to illustrate through CRLB derivations that the proposed TR range estimator (unlike the conventional estimator) uses multipath to its advantage. The TR/GPR range estimator does not make any dispersion-free assumption; therefore, the proposed estimator is extendible to a dispersive environment. For further discussion on the effects of dispersion on GPR, the reader is referred to [17].

The organization of this paper is as follows. Section II provides a review of the receiver used in the GPR applications for estimating the target's range and formulates the problem for both conventional and TR-based range estimators. Section III derives the CRLBs as the variances of the estimated target's range obtained from the conventional and TR/GPR estimators. Section IV compares the performances of the two systems in an electromagnetic simulation environment based on the FDTD model. Finally, Section V concludes this paper.

\section{SySTEM MODEL}

The range $R$ of an unknown stationary target is estimated from the round-trip time delay $\tau$ for the probing waveform to travel out to the target and back to the GPR. Expressed in terms of the root MSE $\sigma_{\tau}$ for the time delay, the root MSE $\sigma_{R}$ for the range (for an unbiased estimator) is given by $\sigma_{R}=(c / 2) \sigma_{\tau}$, where $c$ is the propagation velocity. As such, the range-estimation problem is equivalent to accurately estimating the time delay $\tau$ between the transmitted and received signals.

Fig. 2(a) shows the conventional range-estimation approach, where the input $\tilde{y}(t)$ to the MF is the signal reflected from the 
target including the observation noise $v(t)$. The received GPR signal contains several delayed and attenuated copies of the transmitted signal $f(t)$. The MF correlates the received signal with the waveform of the originally transmitted signal $f(t)$. Time delay $\tau$ (corresponding to the radar range) is derived from the output of the complex envelope detector $z(t)$ at which $z(t)$ assumes its maximum value. Fig. 2(b) shows our proposed TR MF estimator. The TR/GPR estimator uses the conventional GPR estimator to obtain $\tilde{Y}(\omega)$, the Fourier transform of the conventional range estimator observation $\tilde{y}(t)$. Then, this signal is time reversed, energy normalized, and used for TR probing. Mathematically, the TR probing signal is given by $k \tilde{Y}^{*}(\omega)$, with $*$ denoting the complex conjugate operation, and its backscatter observed after the TR step is denoted by $\tilde{X}(\omega)$. The receiver, shown in Fig. 2(b), correlates the received signal $\tilde{X}(\omega)$ with the waveform of the time-reversed transmitted signal, and the time delay $\tau_{\mathrm{TR}}$ is derived from the maximum output of the TR complex envelope detector. Throughout this paper, we use the complex envelope notation in our analysis to account for the carrier frequency so that the baseband signal $f(t)$ takes the form $\Re\left\{\tilde{f}(t) e^{j \omega_{0} t}\right\}$ after modulation, where $\tilde{f}(t)$ is the complex envelope of the signal $f(t)$ and $\omega_{0}=2 \pi f_{0}$, with $f_{0}$ being the carrier frequency. Notation $\Re\{\cdot\}$ refers to the real component of the complex variable included within the curly parentheses. To further illustrate the working of Fig. 2(a), we consider a direct-path model (based solely on Path D in Fig. 1) for which the complex envelope of the signal at the system's input of the MF in the conventional range estimator is given by

$$
\tilde{y}^{(1)}(t)=A_{1} f(t-\tau)+v(t)
$$

where $A_{1}=\left|A_{1}\right| e^{j \psi_{1}}$ denotes direct-path attenuation with magnitude $A_{1}$ and phase $\psi_{1}$. The measurement noise $v(t)$ is assumed to be Gaussian and white with power spectral density of $N_{0}$ within the frequencies of interest $[-B / 2, B / 2]$, where $B$ is the bandwidth of the signal. Consequently, the variance $\eta_{0}$ of the noise within the desired frequency range is $N_{0} B$. The complex envelope of the output of the MF is

$$
\tilde{z}^{(1)}(t)=\int_{-\infty}^{+\infty} h(\lambda-t) \tilde{y}^{(1)}(\lambda) d \lambda
$$

where $h(t)=f(-t)$ is the impulse response of the MF. The square-law detector estimates the delay by solving the following optimization problem

$$
\tau_{e}=\max _{\tau}\left\{\left|\tilde{z}^{(1)}(\tau)\right|^{2}\right\}
$$

where $\tau_{e}$ denotes the estimate of the true delay $\tau$. The conventional range estimator implemented in Fig. 2(a) follows the approach proposed by Knapp and Carter [18], who showed that the optimal maximum-likelihood delay estimator for stationary signals with large time bandwidth product is essentially a crosscorrelator or an MF.

In the following analysis, we assume a two-path propagation model to derive analytic expressions for the variances of the delay error in both the conventional and TR estimators. The results for the two-path model can be generalized to include additional multipaths. Furthermore, we assume that most of the clutter interference in the received signal is compensated using the background subtraction procedure. Our focus is primarily on the residue target response, which contains both the direct and secondary paths. The proposed TR/GPR range estimator is based on the following steps.

1) Forward Probing: The channel is probed with the modulated signal $f(t) e^{j \omega_{0} t}$. For the two-path model, the complex envelope of the GPR received signal is

$$
\tilde{y}^{(2)}(t)=A_{1} f(t-\tau)+A_{2} f(t-\tau-\Delta \tau)+v(t)
$$

where $A_{2}=\left|A_{2}\right| e^{j \psi_{2}}$ is the attenuation of the second path and contains the complex phase $\psi_{2}$. The term $(\tau+\Delta \tau)$ is the delay introduced by the second path. Applying the Fourier transform (FT) to (4), we get

$$
\tilde{Y}^{(2)}(\omega)=\underbrace{\left(A_{1}+A_{2} e^{-j \omega \Delta \tau}\right)}_{G(\omega)} e^{-j \omega \tau} F(\omega)+V(w)
$$

where the channel frequency response because of the multipath is $G(\omega)=\left(A_{1}+A_{2} e^{-j \omega \Delta \tau}\right)$. With reference to Fig. 1, the second path (Path M2) originates from the clutter and produces the second component $A_{2} f(t-\tau-\Delta \tau)$ in (4). Unlike the direct path (Path D) and reflective path from the surface (Path $G$ ) shown in Fig. 1, which are eliminated by the background subtraction procedure, Path M2 includes interactions between the clutter and target. The interactions remain even after background subtraction and are modeled as the second component in (4).

2) MF Estimation: The conventional approach for range estimation uses (4) to estimate the round time delay $\tau_{\text {conv }}$ of the signal reflected from the target. Note that the transfer function of the MF for the conventional approach is $H_{\text {ref }}(\omega)=F^{*}(\omega)$, where $F(\omega)$ is the FT for $f(t)$ and operation $*$ denotes conjugation.

3) TR Probing: As shown in Fig. 2(b), the received signal in (4) is time reversed (equivalent to phase conjugation in the frequency domain), energy normalized, and retransmitted to probe the channel a second time. The complex envelope of the time-reversed signal is given by $k \tilde{Y}^{(2)^{*}}(\omega)$, where $k$ is an energy normalization factor. The complex envelope of the received TR signal in the frequency domain is given by

$$
\tilde{X}^{(2)}(\omega)=\underbrace{k F^{*}(\omega)|G(\omega)|^{2} e^{-j \omega \tau}}_{\tilde{X}^{\mathrm{TR}}(\omega)}+W(\omega)
$$

where $W(\omega)$ represents the accumulated observation noise. The inverse FT of the signal component $\tilde{X}^{\mathrm{TR}}(\omega)$ of $\tilde{X}^{(2)}(\omega)$ for a two-path propagation model in (6) is given by

$$
\begin{aligned}
\tilde{x}^{(\mathrm{TR})}(t) & =k\left[\left(\left|A_{1}\right|^{2}+\left|A_{2}\right|^{2}\right) f(t-\tau)\right. \\
+ & \left.A_{1} A_{2}^{*} f(t-\tau+\Delta \tau)+A_{1}^{*} A_{2} f(t-\tau-\Delta \tau)\right]
\end{aligned}
$$


We use (7) in Section III to evaluate the variance of the time-delay error in the TR MF estimator.

4) TR MF Estimation: The TR approach is based on correlating the backscatter $\tilde{x}^{(2)}(t)$ of the TR signal observed in Step 3) with the TR probing signal $k \tilde{y}^{(2)}(-t)$. In the frequency domain, this is equivalent to applying an MF with the transfer function

$$
\begin{aligned}
H_{\mathrm{ref}}^{\mathrm{TR}}(\omega) & =\left(k \tilde{Y}^{(2)^{*}}(\omega)\right)^{*} \\
& =k \tilde{Y}^{(2)}(\omega)
\end{aligned}
$$

to the TR observation $\tilde{X}^{(2)}(\omega)$. The complex envelope of the output of the MF for the TR estimator becomes

$$
\tilde{R}^{(2)}(\omega)=\tilde{X}^{(2)}(\omega) H_{\mathrm{ref}}^{\mathrm{TR}}(\omega)
$$

and the estimation of delay is obtained from

$$
\hat{\tau}_{\mathrm{TR}}=\max _{\tau}\left\{\left|\tilde{r}^{(2)}(\tau)\right|^{2}\right\}
$$

where waveform $\tilde{r}^{(2)}(t)$ is the inverse FT of $\tilde{R}^{(2)}(\omega)$. Note that (4) and (7) are based on a simplified two-path propagation model suitable for the CRLB analysis and the actual observations will be more complicated with a rich multipath component.

In the following section, we compare the accuracy of our proposed TR-based algorithm [Steps 3) to 4)] with that of the conventional algorithm, which consists of Steps 1) and 2).

\section{CRAmér RAo Lower Bounds}

Result 1 quantifies the theoretical accuracy of the estimated value of the range obtained from the conventional GPR system for the direct-path model (1). In our CRLB analysis, the multipath attenuation factors $A_{1}$ and $A_{2}$ are assumed known and complex. Furthermore, the time delay is deterministic, as is commonly assumed in the GPR applications. The proof of the result is included in the Appendix at the end of this paper.

Result 1: The error variance of GPR time-delay measurement for a direct-path model (1) based on either the conventional or TR range estimator is the same and is given by

$$
\operatorname{var}\left\{\sigma_{\hat{\tau}}^{(1)}\right\} \geq \frac{2 \pi}{\left(\left|A_{1}\right|^{2} / N_{0}\right) \beta_{1}^{2}}
$$

where

$$
\beta_{1}^{2}=\int_{-\infty}^{+\infty} \omega^{2}|F(\omega)|^{2} d \omega
$$

and $N_{0}=\eta_{0} / B$ is the noise power per unit bandwidth with $B$ being the bandwidth of the probing signal $f(t)$.

Result 2 extends the range accuracy to the two-path model (4). As for Result 1, the proof of Result 2 is included in the Appendix of this paper.
Result 2: The error variance of GPR time-delay measurement for a two-path model (4) using the conventional range estimator is given by

$\operatorname{var}\left\{\sigma_{\hat{\tau}}^{(2)}\right\} \geq \frac{2 \pi}{\left(1 / N_{0}\right)\left[\left(\left|A_{1}\right|^{2}+\left|A_{2}\right|^{2}\right) \beta_{1}^{2}+2 \Re\left\{A_{1}^{*} A_{2}\right\} \beta_{\Delta \tau}^{2}\right]}$

where

$$
\beta_{\Delta \tau}^{2}=\int_{-\infty}^{+\infty} \omega^{2} \cos (\omega \Delta \tau)|F(\omega)|^{2} d \omega
$$

Note that the variance for the direct-path model (Result 1) is a special case of Result 2 with $A_{2}=0$. Result 3 derives the error variance for the delay estimated using the TR approach based on the two-path model. The proof for Result 3 is included in the Appendix.

Result 3: The error variance of GPR time-delay measurement for a TR estimator based on a two-path model (7) is given by

$$
\begin{aligned}
\operatorname{var}\left\{\sigma_{\hat{\tau}}^{(2) \mathrm{TR}}\right\} \geq & \left(2 \pi N_{0} / k^{2}\right) \\
\times & \left\{\left[\left(\left|A_{1}\right|^{4}+\left|A_{2}\right|^{4}+4\left|A_{1}\right|^{2}\left|A_{2}\right|^{2}\right) \beta_{1}^{2}\right.\right. \\
& +4 \Re\left\{A_{1}^{*} A_{2}\right\}\left(\left|A_{1}\right|^{2}+\left|A_{2}\right|^{2}\right) \beta_{\Delta \tau}^{2} \\
& \left.\left.+2 \Re\left\{\left(A_{1}^{*}\right)^{2}\left(A_{2}\right)^{2}\right\} \beta_{2 \Delta \tau}^{2}\right]\right\}^{-1}
\end{aligned}
$$

where

$$
\beta_{m \Delta \tau}^{2}=\int_{-\infty}^{+\infty} \omega^{2} \cos (m \omega \Delta \tau)|F(\omega)|^{2} d \omega, \quad \text { for } m=1,2
$$

Note that Result 3 simplifies to Result 1 if the second multipath does not exist $\left(A_{2}=0\right)$ within a scaling factor of $\left(k\left|A_{1}\right|\right)^{2}$ that accounts for the energy normalization step introduced in (6) by TR.

Comparing Results 2 and 3, it is straightforward to show that the error variance for the TR range estimator is smaller than that of the conventional estimator. In the TR estimator, the effect of the multipath is considered in the TR probing signal $G^{*}(\omega) F^{*}(\omega)$, where $G(\omega)$ models the multipath. In the conventional approach, the effect of multipath is not considered. Consequently, the TR estimator is more accurate than the conventional range estimator.

As a final note to our discussion, we observe that if the time delay $\tau$ is modeled as a random variable (as is normally done in the context of digital communication systems), then the noise variance $\eta_{0}$ in the CRLB is replaced by the variance of the received signal used at the input of the estimator. This leads to the results proved by Jin et al. [12]. 


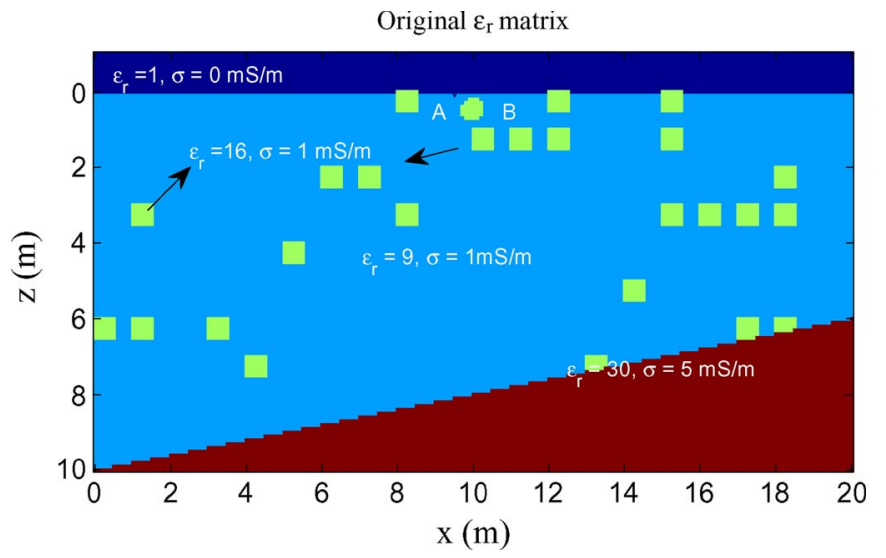

Fig. 3. Experimental domain specifying the electrical properties for the drysoil TM-mode FDTD simulations presented in this paper. The symbol 0 represents the target, while the symbol $\square$ represents clutter. For humid soil, the conductivity values for the soil are changed to $150 \mathrm{mS} / \mathrm{m}$.

\section{Simulation Results}

In this section, we investigate the performance of our proposed range estimation through a 2-D FDTD model for the GPR. To begin, we introduce the GPR model for a basic surface reflection and its transverse-magnetic (TM)-mode FDTD implementation in Section IV-A. Then, Section IV-B uses the FDTD-simulated GPR model as a test case for the conventional MF estimator. Finally, Section IV-C presents our proposed TR range-estimation algorithm using the GPR model and compares it with the conventional MF range estimation.

\section{A. FDTD Simulation Environment}

Fig. 3 shows a 2-D $(11 \mathrm{~m} \times 20 \mathrm{~m})$ multilayered spatial domain highlighting the electrical properties of different layers used to test the GPR system. When used for underground object detection, most GPR devices rely on the difference between the dielectric properties of the underground object and its surrounding soil. The subsurface consists of two layers separated by a dipping boundary with air at the top. For most geologic materials, the dielectric constant $\epsilon_{r}$ lies within a range of 3-30 with soil at the lower end of this range at about 5-10. Nonmetallic objects such as plastic antitank landmines have dielectric constants within a range of about 3-10 depending on their composition, while metallic objects have much higher values (almost close to infinity) since these are strong conductors of electricity. The values for the dielectric constants and the conductivities used in our simulation are as follows. The upper layer, representative of soil, has a relative dielectric constant of $\epsilon_{r}=9$ and a conductivity of $\sigma=1 \mathrm{mS} / \mathrm{m}$. The lower layer, representative of material in the saturated zone, has $\epsilon_{r}=30$ and $\sigma=5 \mathrm{mS} / \mathrm{m}$. Within the upper layer, there is one anomalous circle, representative of a target buried beneath the earth's surface, that has $\epsilon_{r}=16$ and $\sigma=1 \mathrm{mS} / \mathrm{m}$ and is located at a depth of $0.5 \mathrm{~m}$ below the earth. Then, the target range will be within $0.7 \mathrm{~m} \leq r \leq 0.8 \mathrm{~m}$ considering the size of the target. The square blocks, shown in the figure, represent randomly distributed clutters with the same electrical properties as the target. An air-earth interface is shown in the model at $z=0$;

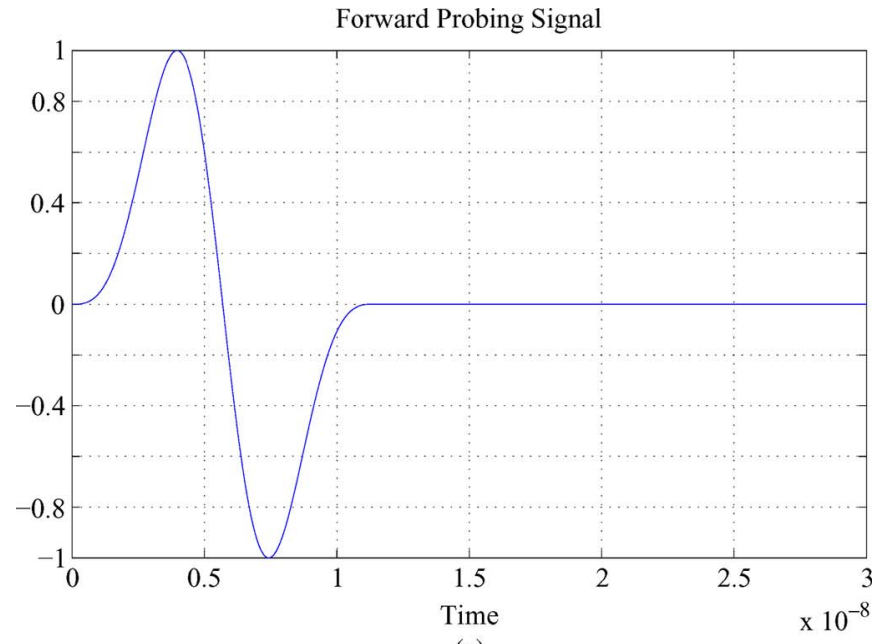

(a)

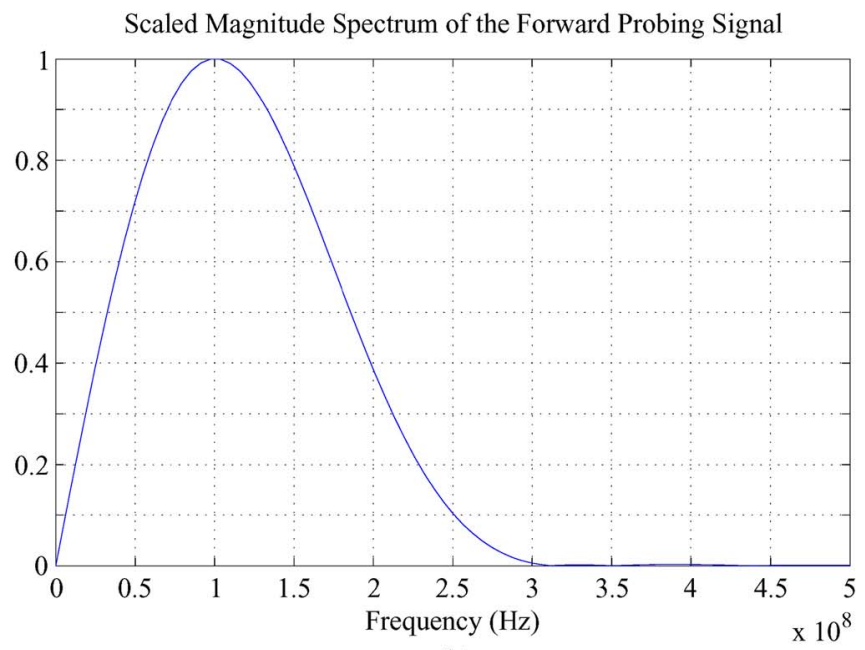

(b)

Fig. 4. Normalized first derivative of the Blackman-Harris pulse used as the forward probing signal in our simulations. (a) Time-domain and (b) frequencydomain representations. Subplot (b) shows that the dominant frequency of the probing pulse (where the magnitude spectrum is maximum) equals $100 \mathrm{MHz}$.

this is performed by simply adding a thin upper layer with $\epsilon_{r}=$ 1 and $\sigma=0$ to the grid. For all materials, the permittivity $\mu$ was set equal to its free-space value $\mu_{0}=1.25 \times 10^{-6}$. The GPR transmitter and receiver (labeled as $\mathrm{A}$ and $\mathrm{B}$, respectively) are located along the air-earth interface at horizontal coordinates $x=9.5 \mathrm{~m}$ and $x=10.5 \mathrm{~m}$, respectively. For conventional range estimation, the source pulse used in our simulations is the normalized first derivative of a Blackman-Harris window used commonly in geophysical FDTD modeling [19]. Fig. 4 shows the probing pulse and its magnitude spectrum with a dominant frequency of $100 \mathrm{MHz}$, which is fed as the field component $E_{y}$ at the source location in our TM-mode simulation. The resulting pulse after propagating through the grid recorded at the receiver location is used by the conventional MF estimator. Because our FDTD simulation is based on a discretized 2-D model, the transmitter and the receiver are actually line elements, extending to positive and negative infinities in the dimension perpendicular to the grid plane. An important step in the FDTD modeling is choosing appropriate time and spatial discretization intervals for the simulations. To control numerical dispersion, 


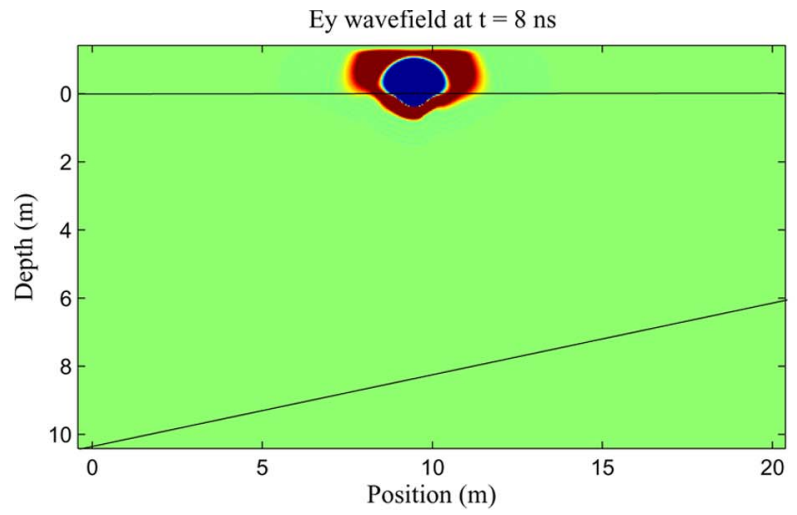

(a)

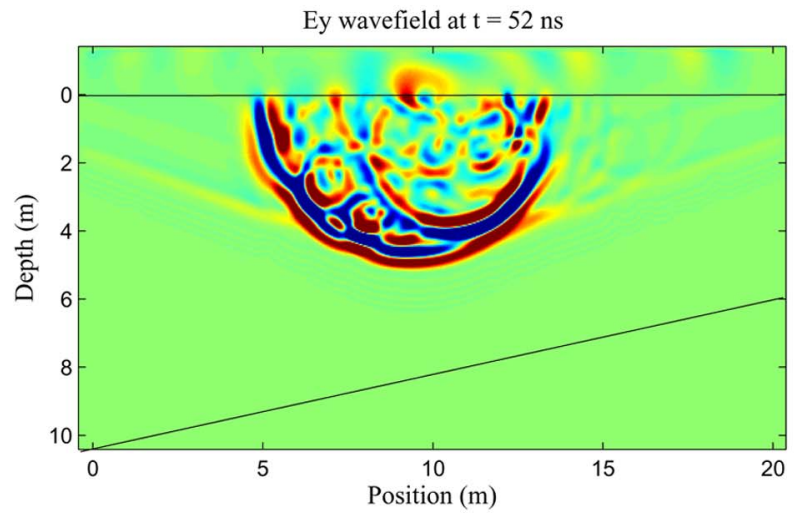

(c)

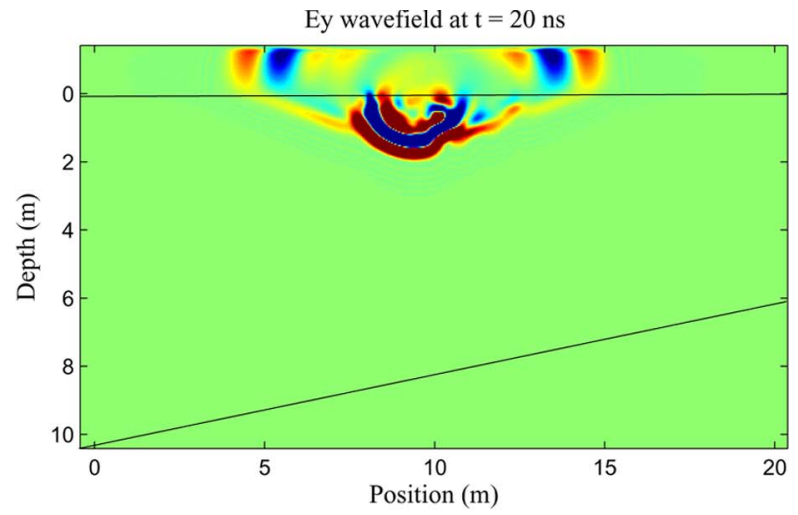

(b)

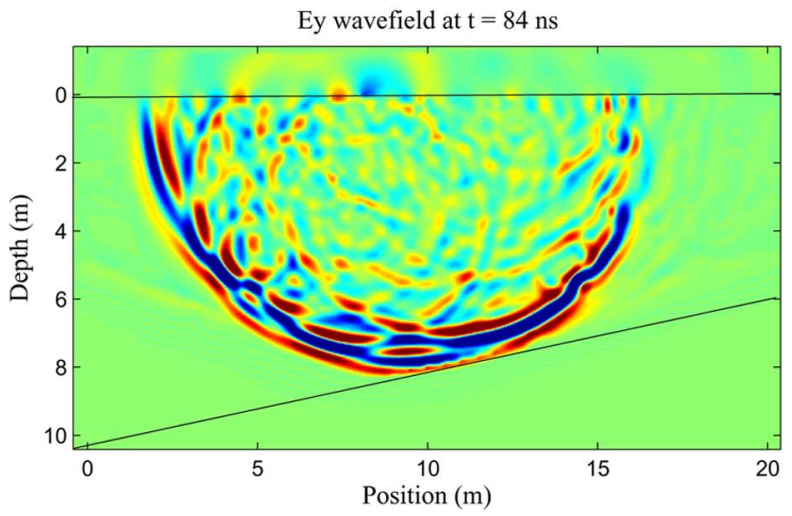

(d)

Fig. 5. Snapshots of the $E_{y}$ component of the electrical wavefield propagating through the experimental domain (Fig. 3) in the TM-mode FDTD simulation at time instants of (a) 8, (b) 20, (c) 52 , and (d) $84 \mathrm{~ns}$.

the FDTD scheme that we used allows for five samples per minimum wavelength. This leads to the spatial discretization grid with $\Delta x=\Delta z=0.04 \mathrm{~m}$. In order for the FDTD scheme to remain numerically stable, we choose $\Delta t$ based on the following bound [19]

$$
\Delta t \leq \frac{6}{7} \sqrt{\frac{\mu_{\min } \epsilon_{\min }}{\left(1 / \Delta x^{2}+1 / \Delta z^{2}\right)}}
$$

where $\mu_{\min }$ and $\epsilon_{\min }$ are the minimum magnetic permeability and dielectric permittivity values, respectively, present in the modeled environment. Our code features perfectly matched layer (PML) absorbing boundaries [20] to avoid reflections from the edges of the modeling grid.

Fig. 5 shows snapshots of the $E_{y}$ field component over the medium at different times during the FDTD simulation for the source located at $x=9.5 \mathrm{~m}$, when excited with the probing signal shown in Fig. 4. At $t=8$ ns [Fig. 5(a)], the wavefield is spreading out from the source before it encounters any heterogeneities within the earth. The head waves show that the field is traveling more rapidly through the air than through the ground. There is no reflection from the top boundary because of the PML absorbing boundaries used in our simulations. At $t=20 \mathrm{~ns}$ [Fig. 5(b)], the electric field has clearly encountered the buried target since it is being reflected back toward Receiver B. At $t=52$ ns [Fig. 5(c)], the energy reflected back from the target has reached the receiver and the complex field represents the high-clutter environment. The electric field approaches the dipping boundary between the top and bottom layers of the earth at $t=84 \mathrm{ns,}$ as shown in Fig. 5(d).

\section{B. Conventional GPR Estimation}

The signal recorded at the receiving antenna (Antenna B) using the aforementioned simulation environment is shown in Fig. 6(a). In order to isolate the target reflection from the overall reflection field [Fig. 6(a)], background subtraction is used. We repeat the experiment twice with no buried target present and when the target is present and the difference provides the reflection from the target. A similar process is used in the real GPR systems, where the system is moved from a position over ground with no buried target to a position over ground with a buried target. The signals recorded at the two different positions are compared to isolate the target reflection. The direct coupled signal and boundary reflections (if any) are nearly the same with and without the target present, and the effects of these signals are eliminated by the previous subtraction. However, the reflections from the other multipaths (i.e., bottom layer reflection) and the interactions between the clutters and target, with and without target present, are not the same. The background subtraction process cannot eliminate these secondary reflections between the clutters and target. Next, the conventional MF estimation is used to locate the depth of the target according to Step 2) of our proposed algorithm in Section II. The output of the MF used in a conventional range estimator at an SNR of $-5 \mathrm{~dB}$ is shown in Fig. 6(b). This figure 


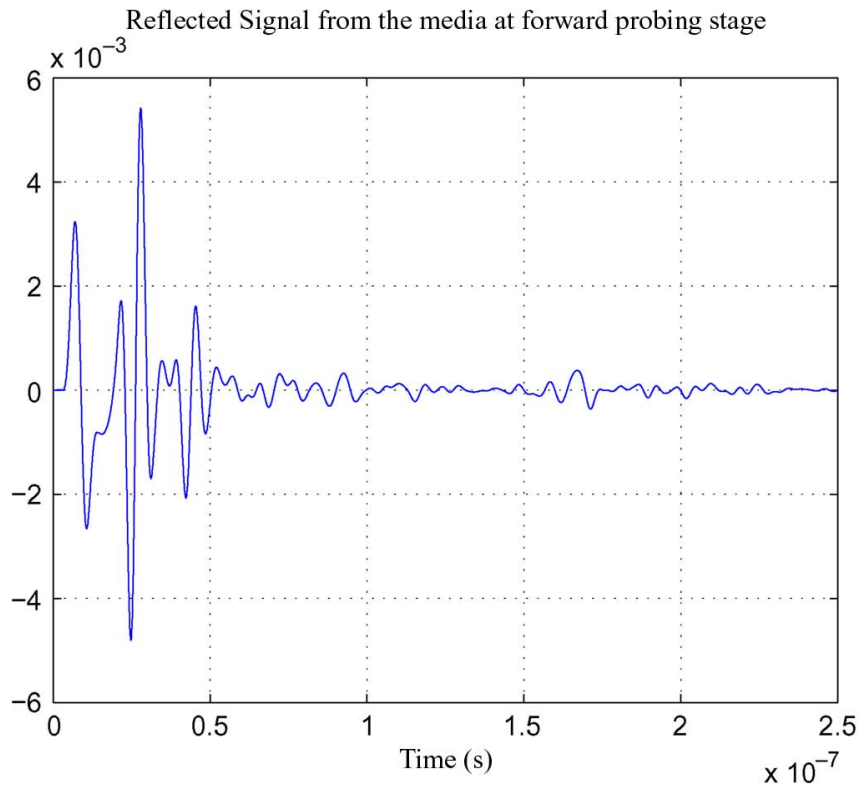

(a)

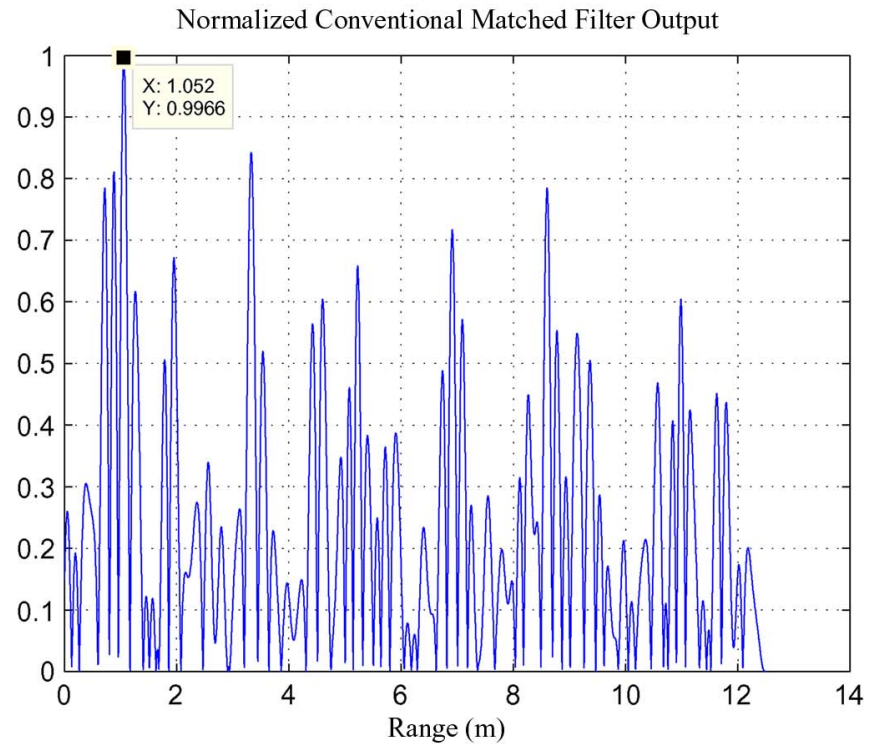

(b)

Fig. 6. (a) Electrical component $E_{y}$ recorded at Receiver B used by the conventional GPR range estimator. (b) Output of the MF used in the conventional range estimator for a buried target (range of $0.7 \mathrm{~m} \leq r \leq 0.8 \mathrm{~m}$ ) at an SNR of $-5 \mathrm{~dB}$.

shows that the peak of the MF output (hence, the estimated target location) occurs at $\hat{r}=1.052 \mathrm{~m}$, while the range of the target is $0.7 \mathrm{~m} \leq r \leq 0.8 \mathrm{~m}$. This error in the GPR estimation is because of the multipath in the environment resulting from strong secondary reflections between the clutters and target. Even with the background subtraction, the conventional MF, when applied to the underground radar data, fails since there are wide fluctuations in the reflected signals due to these secondary interactions and soil inhomogeneities.

\section{TR GPR Estimation}

According to Step 3) of the TR/GPR algorithm (explained in Section II), the received signal shown in Fig. 6 is time reversed, energy normalized, and sent back from Antenna B into the medium. The TR probing signal $k \tilde{y}(-t)$ (which is used as the reference signal in the TR MF) is shown in Fig. 7(a), where the direct-path reflective signal is subtracted using the background subtraction step used in the conventional estimator. Antenna A records the final TR observation $\tilde{x}(t)$, which is shown in Fig. 7(b). Similar to the conventional estimator, we repeat the experiment with and without target and perform the background subtraction in order to derive the target reflection. Then, the TR MF is applied to the TR received signal. Since the reference signal used in the TR/GPR estimator is reshaped to the multipath, we expect the TR MF to provide a more accurate estimation of the range. The output of the TR MF is shown in Fig. 7(c). The TR MF output occurs at $\hat{r}=0.884 \mathrm{~m}$, which is a more accurate estimation than the conventional MF output $(\hat{r}=1.052 \mathrm{~m})$.

Next, we illustrate the superior performance of our proposed TR algorithm by computing the MSEs in estimating the range at different SNRs and comparing them with the MSE resulting from the conventional MF approach. For each SNR, we run a Monte Carlo simulation over 100 independent runs for three different values of conductivities, namely, zero conductivity $(\sigma=0)$ in Fig. 8(a), low conductivity $(\sigma=1 \mathrm{mS} / \mathrm{m})$ in Fig. 8(b), and high conductivity ( $\sigma=150 \mathrm{mS} / \mathrm{m})$ in Fig. 8(c). Plots (a) and (b) correspond to dry soil, while plot (c) corresponds to humid soil. Fig. 8 also shows the analytical CRLBs derived from Results 2 and 3 for the two range estimators based on the simplified two-path model. The attenuation constants $A_{1}$ and $A_{2}$ used to plot the CRLBs are determined by running the forward propagation step in the FDTD simulation twice: first, with only the target present (by removing all sources of clutter in the setup shown in Fig. 3) and, second, with only the clutter source closest to the target present. The backscatters of the probing signal $f(t)$ are recorded at the receiving antenna for the two special cases. Assuming that $A_{1}$ is normalized to one, $A_{2}$ is then approximated by taking the ratio of the energy recorded in the second simulation (with only the clutter source closest to the target present) with respect to the energy recorded in the first simulation (with only the target present). The true value of the estimated delay parameter $\tau$ is estimated by dividing the range of the target with respect to the transmitting/receiving antennas by the average propagation velocity. Similarly, the relative time delay $\Delta \tau$ between the target and its nearest clutter source is estimated by dividing their respective range distances by the average propagation speed and taking the difference. It should also be noted that the estimates for $\left\{A_{1}, A_{2}\right\}$, and $\Delta \tau$ are only needed to plot the CRLB. Both conventional and TR rangeestimation algorithms are based on matched filtering and do not require these estimates to determine the range of the target. The computed value of $\tau$ is used as ground reality or truth in testing the performances of the range estimators. Based on Fig. 8, we make the following observations on the performance of the two range estimators.

1) Comparison between the range estimators: As shown in Fig. 8(a)-(c), the MSE curve for the TR range estimator (denoted by " $\diamond$ ") is lower in value than the MSE curve for the conventional estimator (denoted by " $\triangle$ ") in all cases. 


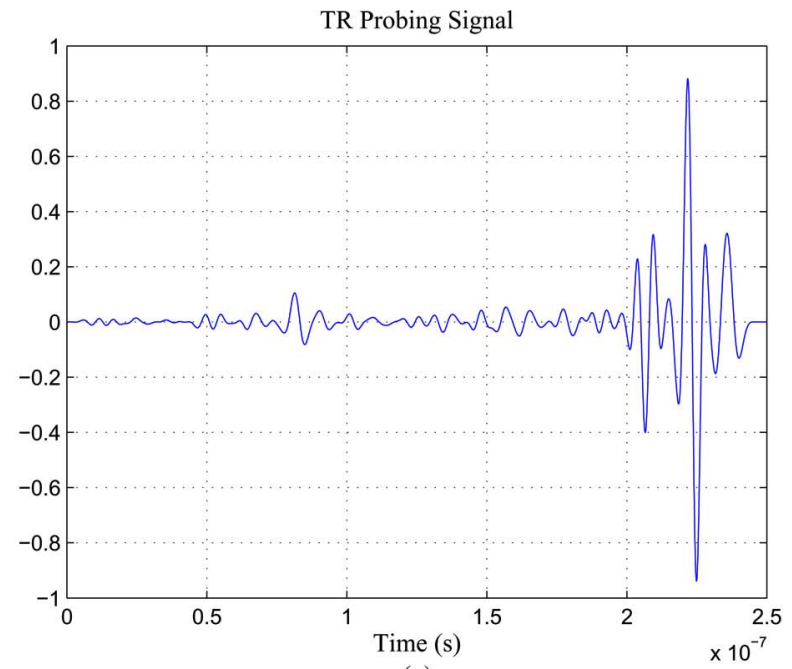

(a)

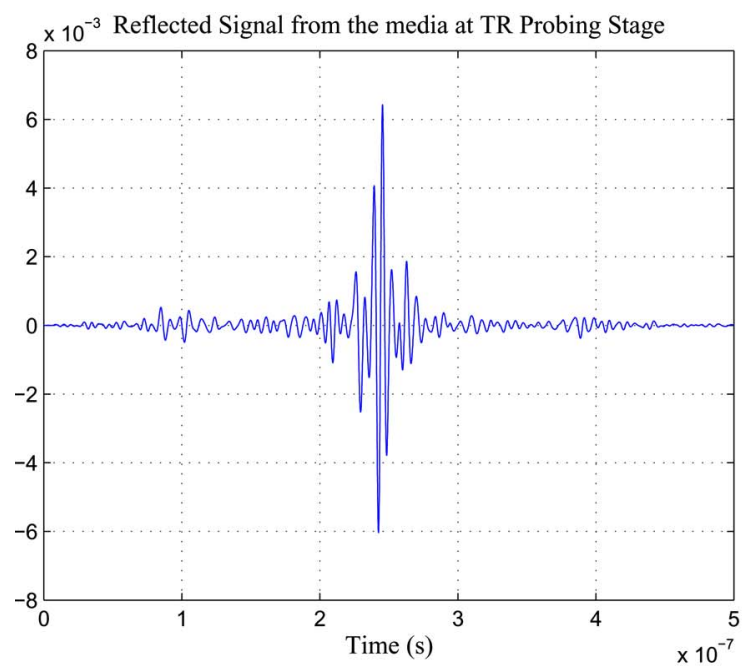

(b)

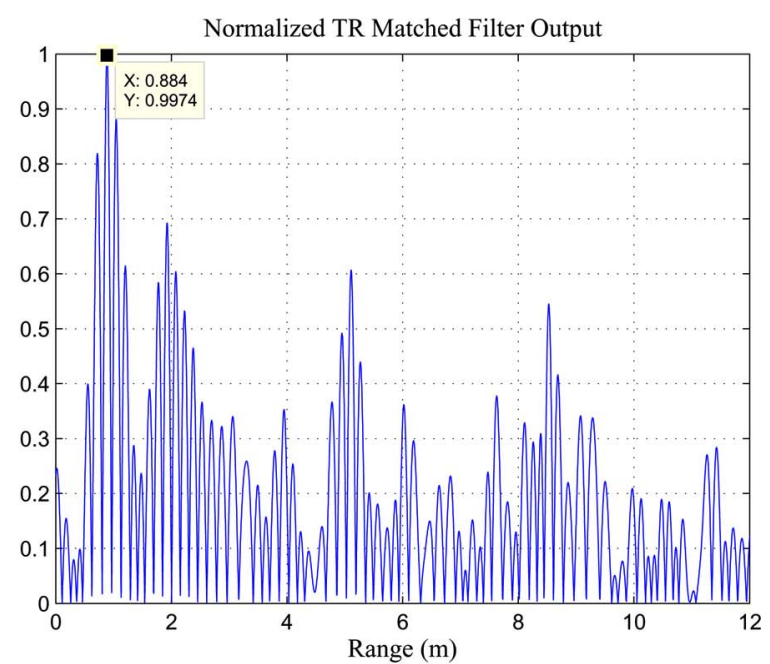

(c)

Fig. 7. (a) TR probing signal obtained by time reversing and energy normalizing the echo received in the forward probing stage. (b) Electrical component $E_{y}$ recorded at Receiver A used by the TR/GPR range estimator. (c) Output of the TR MF for a buried target (range of $0.7 \mathrm{~m} \leq r \leq 0.8 \mathrm{~m}$ ) at an SNR of $-5 \mathrm{~dB}$.

Expressed quantitatively in terms of SNR, the proposed TR/GPR range estimator provides a gain of up to $5 \mathrm{~dB}$ as compared with that of the conventional estimator in dry soil. In other words, the TR/GPR range estimator provides the same MSE for observations with $5 \mathrm{~dB}$ lower SNR. In humid soil, the gain is reduced to $2 \mathrm{~dB}$, which amounts to a significant $60 \%$ reduction in the MSE on a linear scale.

2) Comparison between the CRLBs: In all cases, the CRLBs for the TR/GPR range estimator (denoted by “*”) have lower values than those of the CRLBs for the conventional range estimator (denoted by "o"), which illustrates the potential of obtaining better performance with the TR/GPR range estimator. In humid soil [Fig. 8(c)], the relative value of the attenuation factor $A_{2}$ decreases as compared with the dry soil. Since TR uses multipath to its advantage, therefore, a decrease in $A_{2}$ leads to a reduction in the performance of the TR range estimator, as illustrated in the CRLB shown in Fig. 8(c).

3) Comparison between the performances of the estimators in different types of soils: Lower MSE for zero soil conductivity implies that it is easier to detect the scattered signal from the target in dry soil [Fig. 8(a) and (b)] than in humid soil [Fig. 8(c)]. This supports our observation that the waves that reach the target buried in humid soil are weaker than the waves that penetrate to the same depth in dry soil.

4) MSE difference between CRLBs and actual performances of the range estimators: Although the CRLB for the TR/GPR range estimator has a lower limit in dry soil [Fig. 8(a) and (b)] as compared with that of the CRLB in humid soil [Fig. 8(c)], the actual MSE performance of the TR/GPR estimator also improves in dry soil. Consequently, the difference between the performance curve and the corresponding CRLB is about the same in dry and humid soils. This effect is also demonstrated by Oguz and Gurel [21]. We note that the difference between the CRLBs and the MSEs of the two estimators is due to the coarse modeling of the multipath in the derivations of the CRLBs. The CRLBs are based on a simplified twopath model. In our simulations and also in practice, a multipath is much stronger and results from additional interactions between the target and clutter sources. Since 


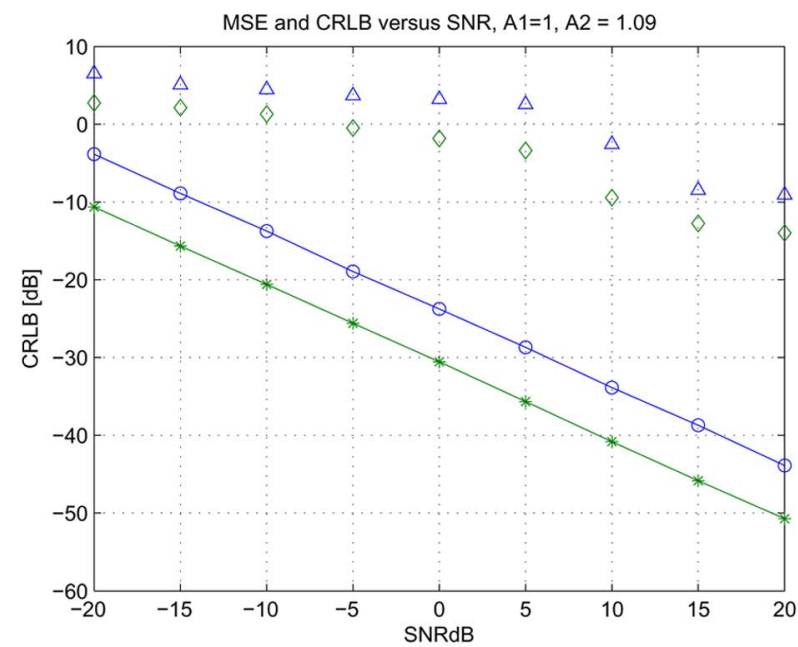

(a)

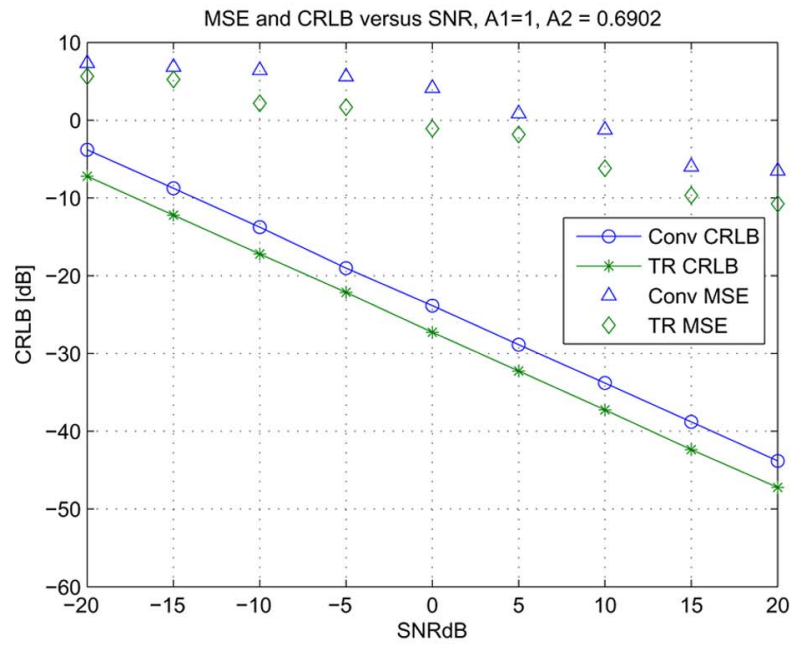

(b)

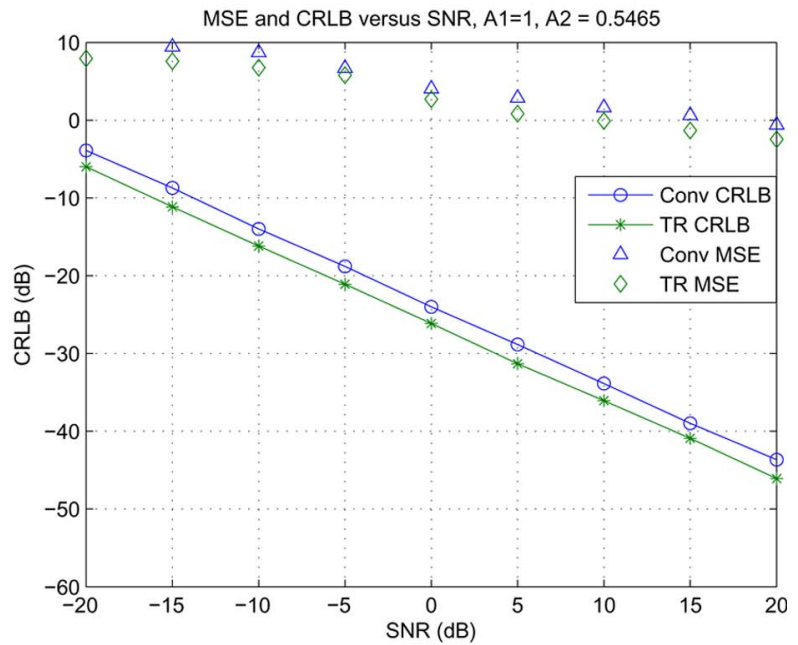

(c)

Fig. 8. MSE and CRLB for both (blue) conventional and (green) TR range estimators for conductivities. (a) $\sigma=0 \mathrm{mS} / \mathrm{m},(\mathrm{b}) \sigma=1 \mathrm{mS} / \mathrm{m}$, and (c) $\sigma=$ $150 \mathrm{mS} / \mathrm{m}$ for the background medium. Plots (a) and (b) represent dry soil, while plot (c) represents humid soil.

the higher order multipath is not adequately modeled in the CRLB derivations, there still remains a difference in the MSEs and their respective CRLBs. The CRLBs for the $N$-multipath model have been presented recently in [23].

As a final note to our discussion, we observe that our simulations are based on the PML absorbing boundary conditions that restrict reflections from the boundaries. To be consistent, both conventional and TR/GPR use the same PML boundary conditions. Using a reflective boundary condition will increase the strength of the multipath. The performance of the conventional range estimator will be adversely affected by increased multipath. Since TR uses multipath to its advantage, the performance of the TR/GPR range estimator is expected to improve under such a condition.

\section{Summary AND Future Work}

In this paper, we proposed a TR-based range estimator to determine the target range in the GPR applications. Through theoretical analysis (based on the CRLB) and numerical simulations, we showed that the accuracy of the TR range estimator is higher than that of the conventional approach particularly in strong scattering environments with rich clutter. While a multipath significantly deteriorates the performance of the conventional range estimator, the performance of the TR estimator remains somewhat unaffected with additional multipaths. We take advantage of the intrinsic ability of TR to adapt the probing waveform to the multipath environment in the proposed TR/GPR range estimator. Expressed quantitatively in terms of SNR, the proposed TR/GPR range estimator provides a gain of up to $5 \mathrm{~dB}$ as compared with the conventional estimator. In other words, the TR/GPR range estimator provides the same for observations with $5 \mathrm{~dB}$ lower SNR. In this paper, our approach was based on a single pair of transmitter and receiver antennas. Since the gain associated with TR improves with an array of antennas, we intend to extend this work to a framework consisting of multiple antenna arrays. The sensitivity of the TR/GPR range estimator to the conductivity of the background medium and some field work validation are other directions likely to be pursued in the near future. 


\section{APPENDIX}

Proof of Result 1: Slepian [22] showed that the variance of the estimated time delay in radar satisfies

$$
\operatorname{var}\left\{\sigma_{\hat{\tau}}^{(1)}\right\} \geq \frac{1}{\left(1 / N_{0}\right) \underbrace{\int_{-\infty}^{+\infty}\left|\frac{\partial}{\partial \tau} A_{1} f(t-\tau)\right|^{2} d t}_{\text {I }}} .
$$

Expressing the FT of $f(t)$ as $F(\omega)$, applying the time shifting property, and taking the partial derivative with respect to $\tau$ gives

$$
\begin{aligned}
\frac{\partial}{\partial \tau}\left\{A_{1} f(t-\tau)\right\} & \stackrel{\mathcal{F}}{\longleftrightarrow} \frac{\partial}{\partial \tau}\left\{A_{1} F(\omega) e^{-j \omega \tau}\right\} \\
& \stackrel{\mathcal{F}}{\longleftrightarrow} A_{1}(-j \omega) F(\omega) e^{-j \omega \tau} .
\end{aligned}
$$

Using Parseval's theorem to solve integral $I$, we get

$$
\begin{aligned}
\mathbf{I} & =\frac{1}{2 \pi} \int_{-\infty}^{+\infty}\left|A_{1}(-j \omega) F(\omega) e^{-j \omega \tau}\right|^{2} d \omega \\
& =\frac{\left|A_{1}\right|^{2}}{2 \pi} \int_{-\infty}^{+\infty} \omega^{2}|F(\omega)|^{2} d \omega
\end{aligned}
$$

which yields Result 1 when simplified.

Proof of Result 2: We use the Slepian inequality (17). For the two-path model, integral $\mathbf{I}$ in (17) changes to

$$
\mathbf{I I}=\int_{-\infty}^{+\infty}\left|\frac{\partial}{\partial \tau}\left[A_{1} f(t-\tau)+A_{2} f(t-\tau-\Delta \tau)\right]\right|^{2} d t
$$

which is expanded as

$$
\begin{aligned}
\mathbf{I I}= & \underbrace{\int_{-\infty}^{+\infty}\left|\frac{\partial}{\partial \tau} A_{1} f(t-\tau)\right|^{2} d t}_{\text {IIA }} \\
& +\underbrace{\int_{-\infty}^{+\infty}\left|\frac{\partial}{\partial \tau} A_{2} f(t-\tau-\Delta \tau)\right|^{2} d t}_{\text {IIB }}+\left(A_{1}^{*} A_{2}+A_{2}^{*} A_{1}\right) \\
& \times \underbrace{\int_{-\infty}^{+\infty}\left[\frac{\partial}{\partial \tau}[f(t-\tau)] \times \frac{\partial}{\partial \tau}[f(t-\tau-\Delta \tau)]\right] d t}_{\text {IIC }}
\end{aligned}
$$

Integrals IIA and IIB are similar to integral $\mathbf{I}$ in (17). Following the same procedure, we get

$$
\mathbf{I I A}=\left|A_{1}\right|^{2} \beta_{1}^{2} /(2 \pi) \quad \mathbf{I I B}=\left|A_{2}\right|^{2} \beta_{1}^{2} /(2 \pi)
$$

Integral IIC is expressed as

$$
\begin{aligned}
\mathbf{I I C}=\frac{2 \Re\left\{A_{1}^{*} A_{2}\right\}}{(2 \pi)^{2}} \iint_{-\infty}^{+\infty}\left(-\omega_{1} \omega_{2}\right) F\left(-\omega_{1}\right) F\left(-\omega_{2}\right) \\
\quad \times e^{j\left(\omega_{1}+\omega_{2}\right) t} e^{-j\left(\omega_{1}+\omega_{2}\right) \tau} e^{j \omega_{2} \Delta \tau} d \omega_{1} d \omega_{2} d t .
\end{aligned}
$$

Changing the order of integration and noting that the innermost integral is given by

$$
\int_{-\infty}^{+\infty} \exp \left(j\left(\omega_{1} \pm \omega_{2}\right) t\right) d t=2 \pi \delta\left(\omega_{1} \pm \omega_{2}\right) .
$$

IIC reduces to

$$
\mathbf{I I C}=\frac{2 \Re\left\{A_{1}^{*} A_{2}\right\}}{(2 \pi)} \int_{-\infty}^{+\infty} \omega^{2}|F(\omega)|^{2} e^{j \Delta \tau \omega} d \omega .
$$

Combining the aforementioned results for IIA, IIB, and IIC proves Result 2.

Proof of Result 3: Using the Slepian inequality, the variance of the estimated time delay for the TR estimator is

$$
\operatorname{var}\left\{\sigma_{\hat{\tau}}^{(2) \mathrm{TR}}\right\} \geq \frac{1}{\left(1 / N_{0}\right) \underbrace{\int_{-\infty}^{+\infty}\left|\frac{\partial}{\partial \tau} x^{(2)}(t)\right|^{2} d t}_{\text {III }} .}
$$

Substituting $x^{(2)}(t)$ from (7), integral III takes the form

$$
\begin{aligned}
\mathbf{I I I}= & k^{2}\left(\left|A_{1}\right|^{2}+\left|A_{2}\right|^{2}\right)^{2} \\
& \times \int\left[\frac{\partial}{\partial \tau} f(t-\tau)\right]^{2} d t+2 k^{2} \Re\left\{\left(A_{1}\right)^{2}\left(A_{2}^{*}\right)^{2}\right\} \\
& \times \int\left[\frac{\partial}{\partial \tau}[f(t-\tau-\Delta \tau) f(t-\tau+\Delta \tau)]\right] d t \\
& \times \int\left[\frac{\partial}{\partial \tau} f(t-\tau)\right]^{2}\left|A_{1}\right|^{2}\left|A_{2}\right|^{2} d t+4 k^{2} \Re\left\{A_{1}^{*} A_{2}\right\}\left(\left|A_{1}\right|^{2}+\left|A_{2}\right|^{2}\right) \\
& \times \int\left[\frac{\partial}{\partial \tau}[f(t-\tau)] \frac{\partial}{\partial \tau}[f(t-\tau+\Delta \tau)]\right] d t .
\end{aligned}
$$

By following the procedure used in Result 2, we can simplify integral III to prove Result 3.

\section{REFERENCES}

[1] E. Pettinelli, A. Di Matteo, E. Mattei, L. Crocco, F. Soldovieri, J. D. Redman, and A. P. Annan, "GPR response from buried pipes: Measurement on field site and tomographic reconstructions," IEEE Trans. Geosci. Remote Sens., vol. 47, no. 8, pp. 2639-2645, Aug. 2009.

[2] G. Borgioli, L. Capineri, P. L. Falorni, S. Matucci, and C. G. Windsor, "The detection of buried pipes from time-of-flight radar data," IEEE Trans. Geosci. Remote Sens., vol. 46, no. 8, pp. 2254-2266, Aug. 2008.

[3] D. Daniels, "A review of GPR for landmine detection," Sens. Imaging: Int. J., vol. 7, no. 3, pp. 90-123, Sep. 2006. 
[4] J. M. Bourgeois and G. S. Smith, "A fully three-dimensional simulation of a ground-penetrating radar: FDTD theory compared with experiment," IEEE Trans. Geosci. Remote Sens., vol. 34, no. 1, pp. 36-44, Jan. 1996.

[5] W. Ng, T. Chan, H. C. So, and K. C. Ho, "Particle filtering based approach for landmine detection using ground penetrating radar," IEEE Trans. Geosci. Remote Sens., vol. 46, no. 11, pp. 3739-3755, Nov. 2008.

[6] C. M. Rappaport, M. El-Shenawee, and H. Zhan, "Suppressing GPR clutter from randomly rough ground surfaces to enhance nonmetallic mine detection," Appl. Subsurface Sens. Technol., vol. 4, no. 4, pp. 311-326, Oct. 2003.

[7] M. Fink, D. Cassereau, A. Derode, C. Prada, P. Roux, M. Tanter, J. L. Thomas, and F. Wu, "Time-reversed acoustics," Rep. Prog. Phys., vol. 63, no. 12, pp. 1933-1995, Dec. 2000.

[8] M. Fink, C. Prada, F. Wu, and D. Cassereau, "Self focusing in inhomogeneous media with time reversal acoustic mirrors," Proc. IEEE Ultrason. Symp., vol. 2, pp. 681-686, Oct. 1989.

[9] L. Borcea, G. Papanicolaou, C. Tsogka, and J Berryman, "Imaging and time reversal in random media," Inv. Problems, vol. 18, no. 5, pp. 12471279, Oct. 2002.

[10] F. K. Gruber, E. A. Marengo, and A. J. Devaney, "Time-reversal imaging with multiple signal classification considering multiple scattering between the targets," J. Acoust. Soc. Amer., vol. 115, no. 6, pp. 3042-3047, Jun. 2004.

[11] G. Shi and A. Nehorai, "A relationship between time-reversal imaging and maximum-likelihood scattering estimation," IEEE Trans. Signal Process., vol. 55, no. 9, pp. 4707-4711, Sep. 2007.

[12] Y. Jin, N. O'Donoughue, and J. M. F. Moura, "Position location by time reversal in communication networks," in Proc. IEEE ICASSP, Mar. 31-Apr. 4 2008, pp. 3001-3004.

[13] J. M. F. Moura and Y. Jin, "Detection by time reversal: Single antenna," IEEE Trans. Signal Process., vol. 55, no. 1, pp. 187-201, Jan. 2007.

[14] F. Foroozan and A. Asif, "Time reversal: Algorithms for M-ary target classification using array signal processing," in Proc. IEEE MILCOM, San Diego, CA, Nov. 2008, pp. 1-7.

[15] S. P. Sira, Y. Li, A. Papandreou-Suppappola, D. Morrell, D. Cochran, and M. Rangaswamy, "Waveform-agile sensing for tracking: A review perspective," Proc. IEEE Signal Process. Mag., vol. 26, no. 1, pp. 53-64, Jan. 2009.

[16] A. Taflove and S. C. Hagness, Computational Electrodynamics: The Finite-Difference Time-Domain Method, 3rd ed. Norwood, MA: Artech House, Jun. 2005.

[17] F. L. Teixeira, W. C. Chew, M. Straka, M. L. Oristaglio, and T. Wang, "Finite-difference time-domain simulation of ground penetrating radar on dispersive, inhomogeneous, and conductive soils," IEEE Trans. Geosci. Remote Sens., vol. 36, no. 6, pp. 1928-1937, Nov. 1998.

[18] C. Knapp and G. Carter, "The generalized correlation method for estimation of time delay," IEEE Trans. Acoust., Speech, Signal Process., vol. ASSP-24, no. 4, pp. 320-327, Aug. 1976.

[19] J. Irving and R. Knight, "Numerical modeling of ground-penetrating radar in 2-D using MATLAB," Comput. Geosci., vol. 32, no. 9, pp. 1247-1258, Nov. 2006.

[20] Y. H. Chen, "Application of perfectly matched layers to the transient modeling of subsurface EM problems," Geophysics, vol. 62, no. 6, pp. 1730-1736, Nov./Dec 1997.

[21] U. Oguz and L. Gurel, "Frequency responses of ground-penetrating radars operating over highly lossy grounds," IEEE Trans. Geosci. Remote Sens., vol. 40, no. 6, pp. 1385-1394, Jun. 2002.

[22] D. Slepian, "Estimation of signal parameters in the presence of noise," IRE Trans. Inf. Theory, vol. 3, pp. 68-69, Mar. 1953.

[23] F. Foroozan and A. Asif, "Cramér-Rao lower bound for time reversal range estimators in N-multipath scattering environments," in Proc. IEEE ICASSP, May 14-19, 2010, pp. 3978-3981.

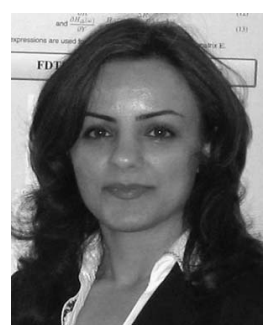

Foroohar Foroozan (S'06) received the B.S. degree in electrical engineering from the Sharif University of Technology, Tehran, Iran, in 1995 and the M.Sc. degree in electrical engineering from Tehran Polytechnic, Tehran, in 1998. She has been working toward the Ph.D. degree in computer science and engineering at York University, Toronto, ON, Canada, since 2005 .

From 1998 to 2005, she was a Senior Research Engineer with the Advanced Information and Communication Technology Research Center, Sharif University of Technology, and also in the semiconductor industry, working on data communication chip design. Her research interests include statistical signal processing, time reversal, and wireless sensor networks.

Ms. Foroozan was the recipient of the postgraduate scholarship from the Natural Science and Engineering Research Council of Canada (2007-2009) and the Ontario Graduate Scholarship award (2006 and 2009).

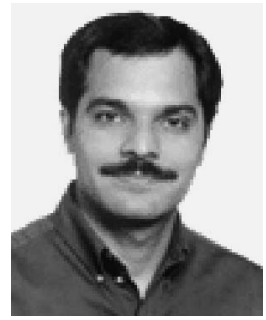

Amir Asif (M'97-SM'02) received the M.Sc. and $\mathrm{Ph} . \mathrm{D}$. degrees in electrical and computer engineering from Carnegie Mellon University (CMU), Pittsburgh, PA, in 1993 and 1996, respectively.

He has been an Associate Professor of computer science and engineering with York University, Toronto, ON, Canada, since 2002. Prior to this, he was with the faculty of CMU, where he was a Research Engineer from 1997 to 1999, and with the Technical University of British Columbia, Vancouver, BC, Canada, where he was an Assistant/ Associate Professor from 1999 to 2002. He has authored over 60 technical contributions, including invited ones, published in international journals and conference proceedings, and a textbook Continuous and Discrete Time Signals and Systems (Cambridge University Press). His current projects include errorresilient scalable video compression, time-reversal array imaging detection, genomic signal processing, and sparse block-banded matrix technologies. $\mathrm{He}$ works in the area of statistical signal processing and communications.

Dr. Asif is a registered Professional Engineer in the province of Ontario. He has been a Technical Associate Editor for the IEEE Signal ProcessING LETTERS (2002-2006 and 2009-present). He has organized two IEEE conferences on signal-processing theory and applications and served on the technical committees of several international conferences. He was the recipient of several distinguishing awards including the CSE Mildred Baptist Teaching Excellence Award from York University's Department of Computer Science and Engineering in 2003 and 2006, the FSE Teaching Excellence Award (Senior Faculty Category) from York University's Faculty of Science and Engineering in 2004 and 2006, the York University Faculty of Graduate Studies' Teaching Award in 2008, and York's University-Wide Teaching Award (Full-Time Senior Faculty Category) in 2008 